Bull. Austral. Math. Soc.

$46 \mathrm{~B} 22,46 \mathrm{~B} 20,58 \mathrm{c} 20$

Vol. 53 (1996) [213-227]

\title{
A SELECTION THEOREM FOR WEAK UPPER SEMI-CONTINUOUS SET-VALUED MAPPINGS
}

\author{
WARREN B. MOORS
}

Let $\Phi$ be a set-valued mapping from a Baire space $T$ into non-empty closed subsets of a Banach space $X$, which is upper semi-continuous with respect to the weak topology on $X$. In this paper, we give a condition on $T$ which is sufficient to ensure that $\Phi$ admits a selection which is norm continuous at each point of a dense and $G_{6}$ subset of $T$. We also derive a variation of James' characterisation of weak compactness, which we use in conjunction with our selection theorem, to deduce some differentiability results for continuous convex functions defined on dual Banach spaces.

\section{INTRODUCTION}

Let $A$ be an open covering of a topological space $T$. A subset $S$ of $T$ is said to be $A$-small if $S$ is contained in a member of $A$. A topological space $T$ is said to be strongly countably complete if there exists a sequence $\left\{A_{n}: n \in \mathbb{N}\right\}$ of open coverings of $T$, with the property that, for any sequence $\left\{F_{n}: n \in \mathbb{N}\right\}$ of non-empty closed subset of $T, \bigcap\left\{F_{n}: n \in \mathbb{N}\right\} \neq \emptyset$ provided $F_{n+1} \subseteq F_{n}$ for all $n \in \mathbb{N}$ and each $F_{n}$ is $A_{n}$-small. It follows immediately from this definition that all locally countably compact and all complete metric spaces are strongly countably complete. In 1974 Isaac Namioka proved the following theorem, [7].

THEOREM 0.1 . Let $T$ be a strongly countably complete regular topological space and let $X$ be a Banach space. If $f: T \rightarrow(X$, weak) is a continuous map, then there is a dense and $G_{\delta}$ subset $G$ of $T$ such that at each point of $G, f$ is norm continuous.

Since this theorem first appeared, it has undergone numerous generalisations, most of which have been aimed at either relaxing the conditions on the domain space to something less stringent than strongly countably complete, or at extending the result to include set-valued mappings. The main result of Section One may be considered to be such a generalisation. Indeed, in Section One, we prove a theorem more general than the following.

\section{Received 1 May 1995}

The Author wishes to express his thanks to the CECM, Simon Fraser University, for their hospitality while the research was being undertaken. The Author also wishes to thank Isaac Namioka for his valuable suggestions concerning the material contained in Section One of the paper.

Copyright Clearance Centre, Inc. Serial-fee code: 0004-9729/96 \$A2.00+0.00. 
TheOREM 0.2. Let $T$ be a regular topological space, which contains as a dense subspace a strongly countably complete space, and let $K$ be a compact Hausdorf space. If $\Phi$ is a set-valued mapping from $T$ into non-empty subsets of $C(K)$, which is upper semi-continuous with respect to the pointwise topology on $C(K)$, then there exists a function $\sigma: T \rightarrow C(K)$ such that $\sigma(t)$ is in the norm closure of $\Phi(t)$ for each $t \in T$, and $\sigma$ is norm continuous at each point of a dense and $G_{\delta}$ subset of $T$.

Section Two is concerned with providing a positive answer to the following question.

Is every continuous convex function $\phi$, defined on the dual of a Banach space $X$, necessarily Fréchet differentiable on a dense and $G_{\delta}$ subset of $X^{*}$ whenever $\partial \phi(f) \cap \widehat{X} \neq$ $\emptyset$ for each $f \in X^{*}$ ?

In answering this question, we prove the following result. If $\phi$ is a continuous convex function defined on the dual of a Banach space $X$, and $\partial \phi(f) \cap \widehat{X} \neq \emptyset$ for each $f \in X^{*}$, then $\partial \phi\left(X^{*}\right) \subseteq \widehat{X}$.

This result may be viewed as an extension of James' characterisation of weak compactness, in the sense, that if $\phi$ is the gauge of the polar of some bounded, closed and convex set $C$ (with $0 \in C$ ), then this result, combined with the Bishop-Phelps theorem, recaptures the fact that $C$ is weak compact.

\section{A SELECTION THEOREM}

The main theorem of this section, Theorem 1.10, has come from an attempt to unify Theorem 2 in $[10]$ and Theorem 5 in [11]. We begin with some definitions. A set-valued mapping $\Phi$ from a topological space $T$ into subsets of a topological space $(X, \tau)$ is said to be $\tau$-upper semi-continuous at a point $t_{0} \in T$ (or $\tau$-usc, for short) if, for each open subset $W$ containing $\Phi\left(t_{0}\right)$, there exists an open neighbourhood $U$ of $t_{0}$ such that $\Phi(U) \equiv \bigcup\{\Phi(t): t \in U\} \subseteq W$. We call a topological space $T$ a Baire space if for each countable family of dense open sets $\left\{O_{n}: n \in \mathbb{N}\right\}$ of $T$, the set $\bigcap\left\{O_{n}: n \in \mathbb{N}\right\}$, is dense in $T$. From this definition one may readily deduce the following facts.

(i) A non-empty open subset of a Baire space is a Baire space.

(ii) If $T$ is a Baire space and $T=\bigcup\left\{F_{n}: n \in \mathbb{N}\right\}$; where each $F_{n}$ is a closed subset of $T$, then $\bigcup\left\{\right.$ int $\left.F_{n}: n \in \mathbb{N}\right\}$ is dense in $T$.

We call a subset of a topological space a $G_{\delta}$ set if it can be expressed as a countable intersection of open sets, and we call a set residual, if it contains, as a subset, the countable intersection of dense open sets. Note that in a Baire space, a subset is residual if, and only if, it contains a dense and $G_{\delta}$ set. In this paper we introduce a new class of Baire spaces, namely the class of $v-\beta$ défavourable spaces, whose definition is amenable to the proof technique considered in Lemma 1.4.

Let $T$ be a topological space. We consider the following $G_{v}$ game played on $T$ between two players $\alpha$ and $\beta$. The player $\beta$ starts by choosing a non-empty open 
subsets $B_{1}$ of $T$. The player $\alpha$ then chosses a non-empty open subset $A_{1} \subseteq B_{1} \subseteq T$ and an element $a_{1} \in T$, (that is, $\alpha$ 's 'move' is the pair $\left(A_{1}, a_{1}\right)$, with $a_{1} \in T$ ). Player $\beta$ responds by selecting a non-empty open subset $B_{2} \subseteq A_{1} \subseteq B_{1} \subseteq T$. Next, $\alpha$ chooses a non-empty open subset $A_{2} \subseteq B_{2} \subseteq A_{1} \subseteq B_{1} \subseteq T$, and an element $a_{2} \in T$. Repeating this procedure infinitely many times, the player $\alpha$ and $\beta$ produce a play. We shall fix the rule that $\alpha$ wins a play if $I \equiv \bigcap\left\{B_{n}: n \in \mathbb{N}\right\} \neq \emptyset$, and either $\operatorname{cl}\left\{a_{k}\right\} \cap I \neq \emptyset$ for some $k \in \mathbb{N}$, or there is a subsequence $\left\{a_{n_{k}}: k \in \mathbb{N}\right\}$ of the sequence $\left\{a_{n}: n \in \mathbb{N}\right\}$, which 'converges' to $I$ (that is, for each open set $W$ with $I \subseteq W$, there exists an $N \cdot \in \mathbb{N}$, such that $a_{n_{k}} \in W$ for each $k>N$ ). Otherwise, the player $\beta$ is said to have won this play. By a strategy $t$ for the player $\beta$ (strategy $s$ for the player $\alpha$ ) we mean a rule which determines each of player $\beta$ 's (player $\alpha$ 's) moves. In particular, for $\beta$, the strategy $t$ determines his first move. In general the $k$ th move of player $\beta$ (player $\alpha$ ) may depend on the previous moves of $\alpha$ (or $\beta$ ), and so we denote the $k$ th move of $\beta$ by $B_{k} \equiv t\left(\left(A_{1}, a_{1}\right), \ldots,\left(A_{k-1}, a_{k-1}\right)\right)(k \geqslant 2)$ ( $k$ th move of $\alpha$ by $\left.\left(A_{k}, a_{k}\right) \equiv s\left(B_{1}, \ldots, B_{k}\right)(k \geqslant 1)\right)$. For notational reasons we denote $\beta$ 's first move by $B_{1} \equiv t\left(\left(\emptyset, a_{0}\right)\right)$, where $a_{0}$ is some fixed element of $T$. We call a strategy $t$ a winning strategy of $\beta$, if $\beta$ wins every play when using the strategy $t$. Likewise, we call a strategy $s$ a winning strategy for $\alpha$, if $\alpha$ wins every play when using the strategy $s$. Furthermore, we call a finite sequence $\left(\left(A_{1}, a_{1}\right), \ldots,\left(A_{n}, a_{n}\right)\right)$ or infinite sequence $\left(\left(A_{1}, a_{1}\right),\left(A_{2}, a_{2}\right), \ldots,\right)$ of pairs, a $t$-sequence if $A_{i} \subseteq t\left(\left(A_{1}, a_{1}\right), \ldots,\left(A_{i-1}, a_{i-1}\right)\right)$ for $2 \leqslant i \leqslant n$ (or for all $i \geqslant 2$ ). and $A_{1} \subseteq t\left(\left(\emptyset, a_{0}\right)\right)$. Similarly we call a finite sequence $\left(B_{1}, \ldots, B_{n}\right)$ or an infinite sequence $\left(B_{1}, B_{2}, \ldots,\right)$ of open sets, an s-sequence if $B_{i} \subseteq s\left(B_{i}, \ldots, B_{i-1}\right)$ for $2 \leqslant i \leqslant n$ (or $i \geqslant 2$ ). Finally, we call a topological space $T$ $v-\beta$ defavourable if there is no winning strategy $t$, for $\beta$ in the $G_{v}$ game played in $T$. We note that it follows from Theorem 1 in [9] that a $v-\beta$ défavourable space is necessarily a Baire space.

For Banach space $X$ with closed unit ball $B(X)$, we shall denote by $\operatorname{ext}\left(B\left(X^{*}\right)\right)$ the set of extreme points of the dual ball $B\left(X^{*}\right)$, and we shall denote by $\sigma_{e}$, the weak topology on $X$ generated by $\operatorname{ext}\left(B\left(X^{*}\right)\right)$. Observe that the $\sigma_{e}$-topology on $X$ is Hausdorff and that the closed unit ball $B(X)$ is also closed in the $\sigma_{\mathrm{e}}$-topology. Both of these observations follow from the fact that for each $x \in X$ there exists an element $f \in \operatorname{ext}\left(B\left(X^{*}\right)\right)$ such that $f(x)=\|x\|$. In the case when $X=C(K),(K$ compact and Hausdorff), and $B(X)$ is the supremum norm ball, the $\sigma_{\mathrm{e}}$-topology is the pointwise topology on $C(K)$. In the case of a Banach space $X$, whose dual ball $B\left(X^{*}\right)$ is rotund, the $\sigma_{e}$-topology is the weak topology on $X$.

We need the following important result, which connects $\sigma_{e}$-compactness to weak compactness. A proof of this result may be found in either $[\mathbf{1}],[\mathbf{6}]$ or $[\mathbf{1 0}]$.

Theorem 1.1. Let $F$ be a bounded subset of a Banach space $X$, which is 
relatively countably compact in the $\sigma_{e}$-topology. Then $F$ is relatively compact in the weak topology.

The key result of this section of the paper is the following consequence of Theorem 1.1. Indeed, this is the very result which enables us to extend Theorem 5 of [11] to include $\sigma_{e}$-upper semi-continuous set-valued mappings.

Corollary 1.2. Let $F$ be an infinite bounded subset of a Banach space $X$. Then there exists a countably infinite subset $E$ of $F$ such that $\sigma_{e}-\operatorname{cl} E \subseteq$ weak-cl $F$.

Proof: Suppose that this is not the case. Then for each countably infinite subset $E$ of $F$ there exists an element $z \in\left(\sigma_{\mathrm{e}}-\operatorname{cl} E\right) \backslash$ weak-cl $F$. Clearly such an element $z$, is a $\sigma_{e}$-cluster point of $E$. Hence by Theorem 1.1, weak- $\operatorname{cl} F$ is weak compact and therefore $\sigma_{\mathrm{e}}$-compact. However, as we remarked earlier, the $\sigma_{\mathrm{e}}$-topology is Hausdorff, and so weak-cl $F$ is $\sigma_{e}$-closed. It now follows that for any countably infinite subset $E$ of $F, \sigma_{e}-\operatorname{cl} E \subseteq$ weak- $\operatorname{cl} F$, which contradicts our initial assumption; and the result follows.

For an element $x$ of a Banach space $X$, and a real number $r>0$, we denote by $B[x, r]$, the set $\{y \in X:\|x-y\| \leqslant r\}$. For a non-empty subset $A$ of $X$ and a real number $r>0$, we shall denote by $B[A, r]$, the set $\bigcup\{B[a, r]: a \in A\}$. Note that if $A$ is $\sigma_{e}$-compact then $B[A, r]$ is $\sigma_{e}$-closed.

In order to simplify the statements of the following results we introduce the following definition. We say that the set-valued mapping $\Phi$ from a topological space $T$ into non-empty subsets of a Banach space $X$ is partially $\sigma_{e}$-upper semi-continuous at a point $t_{0} \in T$ (or partially $\sigma_{e}$-usc, for short), if for each $\sigma_{e}$-closed and bounded subset $C$ of $X$ there exists an open neighbourhood $U$ of $t_{0}$ such that $\Phi(U) \cap C=\emptyset$, whenever $\operatorname{dist}\left(\Phi\left(t_{0}\right), \overline{c o} C\right)>0$. Of course all $\sigma_{e}$-usc set-valued mappings are partially $\sigma_{\mathrm{e}}$-usc.

LEMMA 1.3 . Let $\Phi$ be a partially $\sigma_{e}$-usc set-valued mapping from a topological space $T$ into non-empty subsets of a Banach space $X$. Suppose that for some totally bounded subset $C$ of $X$ and some $\varepsilon>0$ we have that dist $(\Phi(t), B[C, \varepsilon])=0$ for each $t$ in a non-empty open subset $U$ of $T$. Then there exists an element $x \in C$ and a non-empty open subset $V \subseteq U$ such that $\Phi(t) \cap B[x, 2 \varepsilon] \neq \emptyset$ for each $t \in V$.

Proof: Since $C$ is totally bounded there exists a finite set $\left\{x_{j}: 1 \leqslant j \leqslant n\right\} \subseteq$ $C$ such that $B[C, 5 \varepsilon / 4] \subseteq \bigcup\left\{B\left[x_{j}, 3 \varepsilon / 2\right]: 1 \leqslant j \leqslant n\right\}$. Let $F_{j} \equiv\{t \in U: \Phi(t) \cap$ $\left.B\left[x_{j}, 3 \varepsilon / 2\right] \neq \emptyset\right\}$ for each $j \in\{1, \ldots, n\}$. As $\Phi(t) \cap \bigcup\left\{B\left[x_{j}, 3 \varepsilon / 2\right]: 1 \leqslant j \leqslant n\right\} \neq \emptyset$ for each $t \in U$, we have that $U=\bigcup\left\{F_{j}: 1 \leqslant j \leqslant n\right\} \subseteq \bigcup\left\{\operatorname{cl} F_{j}: 1 \leqslant j \leqslant n\right\}$. Hence by a simple induction argument, we see that there exists some $k \in\{1, \ldots, n\}$ such that $\operatorname{int}\left(\mathrm{cl} F_{k}\right) \cap U \neq \emptyset$. The conclusion of the lemma holds for $x \equiv x_{k}$ and $V \equiv \operatorname{int}\left(\operatorname{cl} F_{k}\right) \cap U$.

The next lemma employs a construction considered in [3]. 
Lemma 1.4. Let $\Phi$ be a partially $\sigma_{e}$-usc set-valued mapping from a $v-\beta$ défavourable space $T$ into non-empty subsets of a Banach space $X$. Suppose that for some $\sigma_{\mathrm{e}}$-closed and convex subset $C$ of $X$, and some non-empty open subset $U$ of $T$, we have that $\Phi(t) \cap C \neq \emptyset$ for each $t \in U$. Then for each $\varepsilon>0$ there exists an element $x_{\varepsilon} \in C$ and a non-empty open subset $V_{\varepsilon}$ of $U$ such that $\Phi(t) \cap B\left[x_{\varepsilon}, 3 \varepsilon\right] \neq \emptyset$ for each $t \in V_{\varepsilon}$.

Proof: We consider first the case when $C$ is bounded. In fact, we shall consider the case when $C$ is bounded and $\operatorname{dist}(\Phi(t), C)=0$ for each $t \in U$. Assume, for the purpose of obtaining a contradiction, that for each $x \in C$, int $\{t \in U: \Phi(t) \cap B[x, 3 \varepsilon] \neq$ $\emptyset\}=\emptyset$. From this it follows that int $\{t \in U: \Phi(t) \cap B[x, 2 \varepsilon] \neq \emptyset\}=\emptyset$ for each $x \in$ $B[C, \varepsilon]$. Furthermore, from Lemma 1.3 and the partial $\sigma_{e}$-upper semi-continuity of $\Phi$ we have that for each norm compact subset $D \subseteq B[C, \varepsilon]$, int $\{t \in U: \Phi(t) \cap B[D, \varepsilon]=\emptyset\}$ is a dense open subset of $U$. We define a strategy $t$ for $\beta$ in the $B_{v}$ game played on $T$. First, let $a_{0}$ be a fixed element of $T$, and set $t\left(\left(\theta, a_{0}\right)\right) \equiv U$.

Suppose $\alpha$ chooses $\left(A_{1}, a_{1}\right)$ with $A_{1} \subseteq t\left(\left(\emptyset, a_{0}\right)\right)$ and $a_{1} \in T$. If $a_{1} \in U$, then choose $x_{1} \in \Phi\left(t_{1}\right) \cap B[C, \varepsilon]$ and define $t\left(\left(A_{1}, a_{1}\right)\right) \equiv A_{1} \cap \operatorname{int}\left\{t \in U: \Phi(t) \cap B\left[x_{1}, \varepsilon\right]=\right.$ $\emptyset\}$, otherwise choose $x_{1} \in C$ and define $t\left(\left(A_{1}, a_{1}\right)\right) \equiv A_{1}$. In general, suppose $\alpha$ chooses $\left\{\left(A_{1}, a_{1}\right),\left(A_{2}, a_{2}\right), \ldots,\left(A_{n}, a_{n}\right)\right\}$ so that

$$
A_{n} \subseteq t\left(\left(A_{1}, a_{1}\right), \ldots,\left(A_{n-1}, a_{n-1}\right)\right) \subseteq, \ldots, A_{2} \subseteq t\left(\left(A_{1}, a_{1}\right)\right) \subseteq A_{1} \subseteq t\left(\left(\emptyset, a_{0}\right)\right)
$$

and $\left\{a_{1}, a_{2}, \ldots, a_{n}\right\} \subseteq T$. If $a_{n} \in U$ then choose $x_{n} \in \Phi\left(a_{n}\right) \cap B[C, \varepsilon]$ and define $t\left(\left(A_{1}, a_{1}\right), \ldots,\left(A_{n}, a_{n}\right)\right) \equiv A_{n} \cap \operatorname{int}\left\{t \in U: \Phi(t) \cap B\left[\operatorname{co}\left\{x_{1}, \ldots, x_{n}\right\}, \varepsilon\right]=\emptyset\right\}$, otherwise let $x_{n} \equiv x_{n-1}$ and define $t\left(\left(A_{1}, a_{1}\right), \ldots,\left(A_{n}, a_{n}\right)\right) \equiv A_{n}$. Since $T$ is $v-\beta$ défavourable, $t$ is not a winning strategy for $\beta$ in the game $G_{v}$ played on $T$. Hence in the above, there is a play, with corresponding $t$-sequence $\left\{\left(A_{n}, a_{n}\right): n \in \mathbb{N}\right\}$, in which $\alpha$ wins. Now since $I \equiv \bigcap\left\{A_{n}: n \in \mathbb{N}\right\} \subseteq$ int $\left\{t \in U: \Phi(t) \cap B\left[\operatorname{co}\left\{x_{1}, \ldots, x_{n}\right\}, \varepsilon\right]=\emptyset\right\}$ for each $n \in \mathbb{N}$, it follows that for each $k \in \mathbb{N}, \operatorname{cl}\left\{a_{k}\right\} \cap I=\emptyset$. Hence we may conclude that there is a subsequence $\left\{a_{n_{k}}: k \in \mathbb{N}\right\}$ of $\left\{a_{n}: n \in \mathbb{N}\right\}$ which converges to $I$. Moreover, by Corollary 1.2 we may assume that $\sigma_{e}-\operatorname{cl}\left\{x_{n_{k}}: k \in \mathbb{N}\right\} \subseteq \overline{\operatorname{co}}\left\{x_{n}: n \in \mathbb{N}\right\}$.

Now by the construction, if $t \in I$ then $\Phi(t) \cap B\left[\operatorname{co}\left\{x_{n}: n \in \mathbb{N}\right\}, \varepsilon\right]=\emptyset$ and so $\operatorname{dist}\left(\Phi(t), \overline{c o}\left\{x_{n}: n \in \mathbb{N}\right\}\right)>0$. Therefore by the partial $\sigma_{e}$-upper semi-continuity of $\Phi$ there exists an open set $W$ containing $I$, such that $\Phi(W) \cap \sigma_{e}-\operatorname{cl}\left\{x_{n_{k}}: k \in \mathbb{N}\right\}=\emptyset$. But this is impossible, since the sequence $\left\{a_{n_{k}}: k \in \mathbb{N}\right\}$ converges to $I$, and for $k$ sufficiently large, that is, for $k$ large enough so that $a_{n_{k}} \in U, x_{n_{k}} \in \Phi\left(a_{n_{k}}\right)$. We now consider the case when $C$ is unbounded. From above, it is sufficient to show that for some non-empty open set $V$ of $U$ and some $k \in \mathbb{N}$, dist $\left(\Phi(t), C_{k}\right)=0$ for each $t \in V$, where $C_{k} \equiv C \cap k B(X)$. To this end, suppose that for each $k \in \mathbb{N}$ $F_{k} \equiv\left\{t \in \Phi(t): \operatorname{dist}\left(\Phi(t), C_{k}\right)>0\right\}$ is dense in $U$. From the partial $\sigma_{\mathrm{e}}$-upper semicontinuity of $\Phi$, it follows that for each $k \in \mathbb{N}, U_{k} \equiv \operatorname{int}\left\{t \in U: \Phi(t) \cap C_{k}=0\right\}$ is dense 
in $U$. However, since $U$ is a Baire space with the relative topology, $\bigcap\left\{U_{k}: k \in \mathbb{N}\right\}$ is non-empty (in fact, $\bigcap\left\{U_{k}: k \in \mathbb{N}\right\}$ is dense in $U$ ). But this is impossible, since for each $t \in \bigcap\left\{U_{k}: k \in \mathbb{N}\right\}, \Phi(t) \cap C=\emptyset$. Hence we may conclude that for some $k \in \mathbb{N}$, $F_{k}$ is not dense in $U$ and the result follows.

A function $f$ from a topological space $T$ into a topological space $X$ is called Baire 1 (or of the first Baire class) if the inverse image of each open subset of $X$ is an $F_{\sigma}$ subset of $T$. If $T$ is a metric space and $X$ is a normed linear space, then it is well known that $f$ is Baire 1 if and only if $f$ is the pointwise limit of a sequence of norm continuous functions. Let $\Phi$ be a set-valued mapping from $T$ into subsets of $X$. Then a function $\sigma: T \rightarrow X$ is called a selection of $\Phi$ if $\sigma(t) \in \Phi(t)$ for each $t \in T$. In [11] Stegall proves the following Theorem which gives some very general conditions under which a set-valued mapping from a complete metric space into subsets of Banach admits a selection which is of the first Baire class.

THEOREM 1.5. Suppose that $T$ is a complete metric space, $X$ is a Banach space and $\Phi$ is a set-valued mapping from $T$ into non-empty subsets of $X$ which satisfies the following property:

for each $\varepsilon>0$, each non-empty and closed subset $C$ of $T$ and each closed ball $B$ of $X$, with perhaps infinite radius such that $\Phi(t) \cap B \neq \emptyset$ for all $t \in C$, there exists an open subset $U \subseteq T$ and $V \subseteq X$ such that $U \cap C \neq \emptyset, V \cap B \neq \emptyset$, the diameter of $V$ is less than $\varepsilon$ and $\Phi(t) \cap V \neq \emptyset$ for all $t \in C \cap U$.

Then there exists a Baire 1 function $\sigma: T \rightarrow X$ such that $\sigma(t) \in$ norm- $\operatorname{cl} \Phi(t)$ for each $t \in T$.

We use this result in conjunction with Lemma 1.4 to deduce the following theorem.

ThEOREM 1.6. Suppose $\Phi$ is a partially $\sigma_{e}$-usc set-valued mapping from a complete metric space $T$ into non-empty subsets of a Banach space $X$. Then there exists a Baire 1 function $\sigma: T \rightarrow X$ such that $\sigma(t) \in$ norm-cl $\Phi(t)$ for each $t \in T$.

Proof: From Corollary 1.12 Part (i) (below) we see that a complete metric space is $v-\alpha$ defavourable and from Lemma 1.4 we have that the mapping $\Phi$ satisfies the hypothesis of Theorem 1.5 and so the result follows.

A set-valued mapping $\Phi$ from a topological space $T$ into non-empty subsets of a linear topological space $(X, \tau)$ is called $\tau$-Hausdorff upper semi-continuous at a point $t_{0} \in T$ (or $\tau-H$ usc for short) if for each $\tau$-open neighbourhood $W$ of 0 in $X$, there exists an open neighbourhood $U$ of $t_{0}$ such that $\Phi(U) \subseteq \Phi\left(t_{0}\right)+W$. If $(X, \tau)$ is a locally convex topological space and the set-valued mapping $\Phi$ is $\tau-H$ usc at a point $t_{0} \in T$, then the convex-valued mapping $\Phi^{*}: T \rightarrow 2^{X}$ defined by $\Phi^{*}(t) \equiv \overline{c o} \Phi(t)$, is also $\tau-H$ usc at $t_{0}$. Note that from the 'strong' separation theorem we have that 
a weak- $H$ usc set-valued mapping $\Phi$, from a topological space $T$, whose images are non-empty closed convex subsets of a Banach space $X$, is partially $\sigma_{e}$-usc.

COROLlary 1.7. If $\Phi$ is a weak- $H$ usc set-valued mapping from a complete metric space $T$ into non-empty subsets of a Banach space $X$, then there exists a Baire 1 function $\sigma: T \rightarrow X$ such that $\sigma(t) \in \overline{\operatorname{co}} \Phi(t)$ for each $t \in T$.

Let $X$ be a Banach space. We define the duality mapping $D: S(X) \rightarrow 2^{S\left(X^{*}\right)}$ by $D(x) \equiv\left\{f \in S\left(X^{*}\right): f(x)=1\right\}$. We call a Banach space $X$ an Asplund space, (see [8, p.13] for the original definition) if each separable subspace of $X$ has a separable dual.

Corollary 1.8. Let $X$ be a Banach space and let $B_{1}\left(X^{*}\right)$ be an equivalent, though not necessarily dual, norm on $X^{*}$. Further, let $\sigma_{e}$ be the weak topology on $X^{*}$ generated by ext $\left(B_{1}\left(X^{* *}\right)\right)$. If $X$ can be equivalently renormed so that the graph of the corresponding duality mapping $x \rightarrow D(x)$ contains the graph of a partially $\sigma_{\mathrm{e}}$-usc setvalued mapping, then $X$ is an Asplund space. In particular, if $X$ can be equivalently renormed so that the graph of the corresponding duality mapping $x \rightarrow D(x)$ contains the graph of a weak- $H$ usc set-valued mapping, then $X$ is an Asplund space.

Proof: By Theorem 1.6 the duality mapping $x \rightarrow D(x)$ will admit a Baire 1 selection. The result now follows from Corollary 9 in [11], which states that a Banach space $X$ is an Asplund space if and only if there exists an equivalent norm on $X$ such that the corresponding duality mapping possesses a Baire 1 selection.

As a special case of Corollary 1.8 we may answer Problem 2 of [4].

COROLlary 1.9. If a Banach space $X$ can be equivalently renormed so that the duality mapping $x \rightarrow D(x)$ is weak- $H$ usc on $S(X)$, then $X$ is an Asplund space.

We now present our main selection theorem.

THEOREM 1.10. Suppose that $\Phi$ is a partially $\sigma_{e}$-usc set-valued mapping from a $v-\beta$ défavourable space $T$ into non-empty subsets of a Banach space $X$. Then there exists a function $\sigma: T \rightarrow X$ such that $\sigma(t) \in \operatorname{norm}-\operatorname{cl} \Phi(t)$ for each $t \in T$, and $\sigma$ is norm continuous at each point of a dense and $G_{\delta}$ subset of $T$.

Proof: The proof we present here is essentially the same as that give in [11, Theorem 5]. The proof proceeds by inductively constructing a sequence of functions $\left\{\sigma_{n}: n \in \mathbb{N}\right\}$ which converge uniformly to a function $\sigma$, which will possess the desired selection properties.

STEP 1. Let $\Omega_{1}$ be a maximal family of pairwise disjoint open subsets of $T$ which satisfy the following property:

$*_{1}$ For each $W_{1} \in \Omega_{1}$ there exists an element $x_{W_{1}} \in X$ such that $\Phi(t) \cap B\left[x_{W_{1}}, 1 / 2\right] \neq \emptyset$, for each $t \in W_{1}$. 
Suppose, for the purpose of obtaining a contradiction, that $\mathcal{W}_{1} \equiv \bigcup\left\{W_{1}: W_{1} \in \Omega_{1}\right\}$ is not dense in $T$. Then there exists a non-empty open subset $V \subseteq T$ such that $\mathcal{W}_{1}$ is disjoint from $V$. By Lemma 1.4 (with $C=X$ ) there exists an $x \in X$ and a non-empty open subset $U$ of $V$ such that $\Phi(t) \cap B[x, 1 / 2] \neq \emptyset$ for each $t \in U$. Let $\Omega_{1}^{\prime} \equiv \Omega_{1} \cup\{U\}$. Clearly $\Omega_{1}^{\prime}$ strictly contains $\Omega_{1}$ and satisfies $*_{1}$. However, this contradicts the maximality of $\Omega_{1}$, and hence we may conclude that $\mathcal{W}_{1}$ is dense in $T$. Define $\sigma_{1}: T \rightarrow X$ by, $\sigma(t) \in \Phi(t)$ if $t \in T \backslash \mathcal{W}_{1}$ and $\sigma(t) \equiv x_{W_{1}}$ if $t \in W_{1}$ for some $W_{1} \in \Omega_{1}$. Also, define $\sigma_{0}: T \rightarrow X$ by $\sigma_{0}(t) \equiv \sigma_{1}(t)$ for each $t \in T$. Finally, note that $\sigma_{1}(t) \in B[\Phi(t), 1 / 2]$ for each $t \in T$. Suppose the first $n$ steps of the induction have been completed. Then we shall have a family $\Omega_{n}$ of pairwise disjoint open subsets of $T$ and a function $\sigma_{n}: T \rightarrow X$, such that:

(i) $\mathcal{W}_{n} \equiv \bigcup\left\{W_{n}: W_{n} \in \Omega_{n}\right\}$ is dense in $T$,

(ii) $\sigma_{n}(t) \in \Phi(t)$ for each $t \in T \backslash \mathcal{W}_{n}$, and $\sigma_{n}(t) \equiv x_{W_{n}}$ if $t \in W_{n}$, for some $W_{n} \in \Omega_{n}$.

(Note this means that $\sigma_{n}$ is constant on each $W_{n} \in \Omega_{n}$.)

Furthermore, for each $t \in T, \sigma_{n}(t) \in B\left[\Phi(t), 1 / 2^{n}\right]$ and $\left\|\sigma_{n}(t)-\sigma_{n-1}(t)\right\| \leqslant 1 / 2^{n-1}$.

STEP $n+1$. Let $\Omega_{n+1}$ be a maximal family of pairwise disjoint open subsets of $\mathcal{W}_{n}$ which satisfy the following property:

$*_{n+1}$ For each $W_{n+1} \in \Omega_{n+1}$ there exists a $W_{n} \in \Omega_{n}$ such that $W_{n+1} \subseteq W_{n}$ and there exists an $x_{W_{n+1}} \in X$ with $\left\|x_{W_{n+1}}-x_{W_{n}}\right\| \leqslant 1 / 2^{n}$ such that $\Phi(t) \cap B\left[x_{W_{n+1}}, 1 / 2^{n+1}\right] \neq \emptyset$ for each $t \in W_{n+1}$.

Assume, for the purpose of obtaining a contradiction, that $\mathcal{W}_{n+1} \equiv \cup\left\{W_{n+1}: W_{n+1} \in\right.$ $\left.\Omega_{n+1}\right\}$ is not dense in $T$. Then there exists a non-empty open set $V \subseteq T$ such that $\mathcal{W}_{n+1}$ is disjoint from $V$. Since $\mathcal{W}_{n}$ is dense in $T$, there exists a $W_{n} \in \Omega_{n}$ such that $V \cap W_{n} \neq \emptyset$. Now observe that for each $t \in V \cap W, \Phi(t) \cap B\left[x_{W_{n}}, 1 / 2^{n}\right] \neq \emptyset$. Therefore by Lemma 1.4 there exists a non-empty open subset $U \subseteq V \cap W_{n}$ and an element $x \in B\left[x W_{n}, 1 / 2^{n}\right]$ such that $\Phi(t) \cap B\left[x, 1 / 2^{n+1}\right] \neq \emptyset$ for each $t \in U$. Let $\Omega_{n+1}^{\prime} \equiv \Omega_{n+1} \cup\{U\}$. Clearly $\Omega_{n+1}^{\prime}$ strictly contains $\Omega_{n+1}$ and satisfies $*_{n+1}$. However, this contradicts that maximality of $\Omega_{n+1}$, and hence we may conclude that $\mathcal{W}_{n+1}$ is dense in $T$. We define $\sigma_{n+1}: T \rightarrow X$ as follows. If $t \notin \mathcal{W}_{n}$, then let $\sigma_{n+1}(t) \equiv \sigma_{n}(t)$. If $t \in \mathcal{W}_{n} \backslash \mathcal{W}_{n+1}$, then let $\sigma_{n+1}(t) \in \Phi(t) \cap B\left[\sigma_{n}(t), 1 / 2^{n}\right]$. Finally, if $t \in \mathcal{W}_{n+1}$, then $t \in W_{n+1}$, for some $W_{n+1} \in \Omega_{n+1}$, and we set $\sigma_{n+1}(t) \equiv$ $x_{W_{n+1}}$. Note, for each $t \in T, \sigma_{n+1}(t) \in B\left[\Phi(t), 1 / 2^{n+1}\right]$ and $\left\|\sigma_{n+1}(t)-\sigma_{n}(t)\right\| \leqslant$ $1 / 2^{n}$. It follows from our construction that the sequence $\left\{\sigma_{n}: n \in \mathbb{N}\right\}$ converges uniformly to some function, which we shall call $\sigma$. It is also clear that $\sigma(t) \in$ norm-cl $\Phi(t)$ for each $t \in T$. So to complete the proof it suffices to show that $\sigma$ is norm continuous 
at each point of $\bigcap\left\{\mathcal{W}_{n}: n \in \mathbb{N}\right\}$, which is a dense and $G_{\delta}$ subset of $T$. To this end, let $t_{0} \in \bigcap\left\{\mathcal{W}_{n}: n \in \mathbb{N}\right\}$ and suppose $\varepsilon>0$ is given. Now for each $n \in \mathbb{N}$ and each $t \in T$ we have that $\left\|\sigma_{n+1}(t)-\sigma_{n}(t)\right\| \leqslant 1 / 2^{n}$. Therefore, $\left\|\sigma(t)-\sigma_{n}(t)\right\| \leqslant 1 / 2^{n-1}$ for each $n \in \mathbb{N}$ and $t \in T$. Let us choose $n \in \mathbb{N}$ so that $1 / 2^{n-1}<\varepsilon / 2$, and choose $\dot{W}_{n} \in \Omega_{n}$ so that $t_{0} \in W_{n}$. Then for each $t \in W_{n}$,

$$
\left\|\sigma(t)-\sigma\left(t_{0}\right)\right\| \leqslant\left\|\sigma(t)-\sigma_{n}(t)\right\|+\left\|\sigma_{n}(t)-\sigma_{n}\left(t_{0}\right)\right\|+\left\|\sigma_{n}\left(t_{0}\right)\right\|<\left\|\sigma_{n}(t)-\sigma_{n}\left(t_{0}\right)\right\|+\varepsilon
$$

However, $\sigma_{n}$ is constant on $W_{n}$, and so $\left\|\sigma_{n}(t)-\sigma_{n}\left(t_{0}\right)\right\|=0$. Therefore, for each $t \in W_{n},\left\|\sigma(t)-\sigma\left(t_{0}\right)\right\|<\epsilon$; which completes the proof.

Observe that strictly speaking, Theorem 1.10 is not a selection theorem, unless the images of $\Phi$ are assumed to be closed in the norm topology.

The remainder of this section is devoted to providing some examples of $v-\beta$ défavourable spaces.

We noted earlier that the definition of an $v-\beta$ defavourable space is amenable to the proof technique considered in Lemma 1.4. However a disadvantage with the definition of $v-\beta$ défavourablity is that in general, it is difficult to directly determine whether a given topological space is $v-\beta$ défavourable or not. To overcome this problem we introduce a new class of Baire spaces, contained in the class of $v-\beta$ défavourable spaces whose membership properties are more readily determined.

Let $T$ be a topological space. On $T$ we may consider the following game $G$, (Choquet [2]) played between two players $\alpha$ and $\beta$. As usual, $\beta$ goes first, and chooses a non-empty open subset $B_{1}$ of $T$. Player $\alpha$ then chooses a non-empty open subset $A_{1}$ of $B_{1}$. Player $\beta$ responds to this by choosing a non-empty open subset $B_{2}$ of $A_{1}$. Next, $\alpha$ selects a non-empty open subset $A_{2}$ of $B_{2}$. Continuing this proceedure indefinitely, the players $\alpha$ and $\beta$ produce a play. We declare that $\alpha$ wins a play of the $G$ game if $\bigcap\left\{A_{n}: n \in \mathbb{N}\right\}$ is non-empty, otherwise we declare $\beta$ the winner. In [9], it is proven that on a Baire space there is no winning strategy for $\beta$ in the $G$ game, (in fact, this characterises Baire spaces). We call a topological space $T$ conditionally $v-\alpha$ favourable if $T$ contains a dense set $R$, such that $R$ with the relative topology is a Baire space, and there is a strategy $s$ for $\alpha$ in the $G_{v}$ game played on $T$ such that $\alpha$ wins every play (played according to $s$ ) where the corresponding $s$-sequence $\left\{B_{n}: n \in \mathbb{N}\right\}$ has $\bigcap\left\{B_{n}: n \in \mathbb{N}\right\} \cap R \neq \emptyset$.

Note: it is not a condition of the strategy $s$ that $\bigcap\left\{B_{n}: n \in \mathbb{N}\right\} \cap R$ ever be non-empty.

ThEOREM 1.11. Conditionally $v-\alpha$ favourable spaces are $v-\beta$ défavourable.

Proof: Let $T$ be a conditionally $v-\alpha$ favourable space and let $R$ be the dense subset of $T$ given in the definition of $v-\alpha$ favourable. Further, let $s$ denote the associated strategy on $T$. Consider $\beta$ with a strategy $t$; we shall show that $t$ is not 
a winning strategy for $\beta$ in the $G_{v}$ game played on $T$. We define a strategy $t^{\prime}$ in $\beta$ in the $G$ game played on $R$. For each open set $U$ in $R$ let $U^{*}$ denote the largest open set in $T$ such that $U^{*} \cap R=U$. Clearly if $U_{1} \subseteq U_{2}$ then $U_{1}^{*} \subseteq U_{2}^{*}$. First let $t^{\prime}(\emptyset) \equiv t\left(\left(\emptyset, s_{0}\right)\right) \cap R, s_{0} \in T$, and write $T_{0} \equiv t\left(\left(\emptyset, s_{0}\right)\right)$. If $\alpha$ chooses $A_{1} \subseteq t^{\prime}(\emptyset)$ let $W_{1} \equiv A_{1}^{*} \cap T_{0},\left(S_{1}, s_{1}\right) \equiv s\left(W_{1}\right)$ and $T_{1} \equiv t\left(\left(S_{1}, s_{1}\right)\right)$, then define $t^{\prime}\left(A_{1}\right) \equiv T_{1} \cap R$. (Note: $T_{1} \subseteq S_{1} \subseteq W_{1} \subseteq T_{0}$ ). Inductively, suppose $\alpha$ has chosen $\left\{A_{1}, \ldots, A_{n}\right\}$ so that $A_{n} \subseteq t^{\prime}\left(A_{1}, \ldots, A_{n-1}\right) \subseteq, \ldots, A_{2} \subseteq t^{\prime}\left(A_{1}\right) \subseteq A_{1} \subseteq t^{\prime}(\emptyset)$

At stage $i \quad(1 \leqslant i \leqslant n-1) \beta$ has constructed an open set $W_{i} \subseteq T_{i-1}$ so that $W_{i} \cap R=A_{i}$, and a pair $\left(S_{i}, s_{i}\right)=s\left(W_{1}, \ldots, W_{i}\right)$ and also an open set $T_{i}=$ $t\left(\left(S_{1}, s_{1}\right), \ldots,\left(S_{i}, s_{i}\right)\right)$, in such a way that $\left(\left(S_{1}, s_{1}\right), \ldots,\left(S_{i}, s_{i}\right)\right)$ is a $t$-sequence, $\left(W_{1}, \ldots, W_{i}\right)$ is an $s$-sequence, and $t^{\prime}\left(A_{1}, \ldots, A_{i}\right)=T_{i} \cap R$. (Note: $T_{i} \subseteq S_{i} \subseteq W_{i} \subseteq$ $T_{i-1} \subseteq, \ldots, W_{2} \subseteq T_{1} \subseteq S_{1} \subseteq W_{1} \subseteq T_{0}$.) At stage $n, \beta$ proceeds as follows: Let $W_{n} \equiv A_{n}^{*} \cap T_{n-1},\left(S_{n}, s_{n}\right) \equiv s\left(W_{1}, \ldots, W_{n}\right)$, and $T_{n} \equiv t\left(\left(S_{1}, s_{1}\right), \ldots,\left(S_{n}, s_{n}\right)\right)$. Then $\beta$ sets $t^{\prime}\left(A_{1}, \ldots, A_{n}\right) \equiv T_{n} \cap R$. By construction $\left\{W_{n}: n \in \mathbb{N}\right\}$ is an $s$-sequence and $\left\{\left(S_{n}, s_{n}\right): n \in \mathbb{N}\right\}$ is a $t$-sequence. Since $R$ is a Baire space $t^{\prime}$ is not a winning strategy for $\beta$ in the $G$ game played on $R$. Hence, there is a $t^{\prime}$-sequence $\left\{A_{n}: n \in \mathbb{N}\right\}$ such that $\emptyset \neq \bigcap\left\{A_{n}: n \in \mathbb{N}\right\}=\left\{W_{n}: n \in \mathbb{N}\right\} \cap R$. Since $T$ is conditionally $v-\alpha$ favourable either $\operatorname{cl}\left\{a_{k}\right\} \cap \bigcap\left\{S_{n}: n \in \mathbb{N}\right\} \neq \emptyset$ for some $k \in \mathbb{N}$, or there exists a subsequence $\left\{a_{n_{k}}: k \in \mathbb{N}\right\}$ of $\left\{a_{n}: n \in \mathbb{N}\right\}$ which converges to $\bigcap\left\{S_{n}: n \in \mathbb{N}\right\}$. However, as $\left\{\left(S_{n}, s_{n}\right): n \in \mathbb{N}\right\}$ is a $t$-sequence and $\beta$ does not win this play, the strategy $t$ is not a winning one for $\beta$ in the $G_{v}$ game played on $T$. Therefore $T$ is $v-\beta$ défavourable.

We may now readily obtain some examples of $v-\alpha$ défavourable spaces.

Corollary 1.12. A Baire space $(T, \tau)$ is conditionally $v-\alpha$ favourable and hence $v-\beta$ défavourable if one of the following conditions holds:

(i) $(T, \tau)$ is metrisable;

(ii) $(T, \tau)$ is separable and first countable;

(iii) $(T, \tau)$ is strongly countably complete and regular;

(iv) $T$ is an uncountable set and $\tau$ is the co-finite topology on $T$.

Proof: In all of the following proofs $R=T$.

(i) We define a strategy for $\alpha$ in the following way. If $\beta$ has chosen $\left\{B_{1}, \ldots, B_{n}\right\}$, then define $s\left(B_{1}, \ldots, B_{n}\right) \equiv\left(A_{n}, a_{n}\right)$ where $a_{n} \in A_{n} \subseteq \operatorname{cl} A_{n} \subseteq B_{n}$ and $\operatorname{diam} A_{n}<$ $1 / 2^{n}$. It is easy to see that $\alpha$ wins every play where $\bigcap\left\{B_{n}: n \in \mathbb{N}\right\} \neq \emptyset$.

(ii) Let $\left\{x_{n}: n \in \mathbb{N}\right\}$ be a dense subset of $(T, \tau)$. This time we define the strategy of $\alpha$ as follows. If $C$ has chosen $\left\{B_{1}, \ldots, B_{n}\right\}$, then define $s\left(B_{1}, \ldots, B_{n}\right) \equiv\left(A_{n}, a_{n}\right)$, where $A_{n} \equiv B_{n}$ and $a_{n} \equiv x_{n}$. Now if $I \equiv \bigcap\left\{A_{n}: n \in \mathbb{N}\right\} \neq \emptyset$, then $\operatorname{cl}\left\{a_{n}: n \in \mathbb{N}\right\} \cap I \neq$ $\emptyset$. Let $z \in \operatorname{cl}\left\{a_{n}: n \in \mathbb{N}\right\} \cap I$. Since $z$ has a countable local base either $z \in \operatorname{cl}\left\{a_{n}\right\}$ for some $n \in \mathbb{N}$, or there exists a subsequence $\left\{a_{n_{k}}: k \in \mathbb{N}\right\}$ of $\left\{a_{n}: n \in \mathbb{N}\right\}$ which 
converges to $z$. Hence $\alpha$ wins each play where $\bigcap\left\{A_{n}: n \in \mathbb{N}\right\} \neq \emptyset$.

(iii) Let $\left\{A_{n}: n \in \mathbb{N}\right\}$ be the countable family of open coverings of $T$ given in the definition of strongly countably complete. Again we define a strategy for $\alpha$. Suppose $\beta$ has chosen $\left\{B_{1}, \ldots, B_{n}\right\}$, then define $s\left(B_{1}, \ldots, B_{n}\right) \equiv\left(A_{n}, a_{n}\right)$, where $a_{n} \in A_{n} \subseteq$ cl $A_{n} \subseteq B_{n}$ and $\operatorname{cl} A_{n}$ is $A_{n}$-small. It is easy to see that with this strategy $\alpha$ wins every play.

(iv) In this case we define the strategy $s$ in the following manner. If $\beta$ has chosen $\left\{B_{1}, \ldots, B_{n}\right\}$, then we define $s\left(B_{1}, \ldots, B_{n}\right) \equiv\left(A_{n}, a_{n}\right)$, where $A_{n} \equiv B_{n}$ and $a_{n} \notin\left\{a_{1}, \ldots, a_{n-1}\right\}$. As with (iii), it is easy to see that $\alpha$ wins every play with this strategy.

Proposition 1.13. A topological space $T^{\prime}$ which contains, as a dense subspace, a conditionally $v-\alpha$ favourable space $T$, is conditionally $v-\alpha$ favourable.

Proof: Consider $T$ equipped with the dense subspace $R$ and the strategy $s$ given in the definition of conditionally $v-\alpha$ favourable. Observe that $R$ is dense in $T^{\prime}$ and is a Baire space with a relative topology. For each open set $U$ in $T$, let $U^{*}$ denote the largest open set in $T^{\prime}$ such that $U^{*} \cap T=U$. We shall define a strategy $s^{\prime}$ for $\alpha$ in the $G_{v}$ game played on $T^{\prime}$. First, if $\beta$ chooses $B_{1}$, a non-empty open subset of $T^{\prime}$, we define

$$
s^{\prime}\left(B_{1}\right) \equiv\left(A_{1}^{\prime}, a_{1}^{\prime}\right) \text { where }\left(A_{1}, a_{1}\right)=s\left(B_{1} \cap T\right), A_{1}^{\prime} \equiv A_{1}^{*} \cap B_{1} \text {, and } a_{1}^{\prime} \equiv a_{1} .
$$

In general, suppose $\beta$ chooses $\left\{B_{1}, \ldots, B_{n}\right\}$ so that

$$
B_{n} \subseteq s^{\prime}\left(B_{1}, \ldots, B_{n-1}\right) \subseteq, \ldots, B_{2} \subseteq s^{\prime}\left(B_{1}\right) \subseteq B_{1} .
$$

We define $s^{\prime}\left(B_{1}, \ldots, B_{n}\right) \equiv\left(A_{n}^{\prime}, a_{n}^{\prime}\right)$ where $\left(A_{n}, a_{n}\right)=s\left(B_{1} \cap T, \ldots, B_{n} \cap T\right), A_{n}^{\prime} \equiv$ $A_{n}^{*} \cap B_{n}$, and $a_{n}^{\prime} \equiv a_{n}$ (This makes sense since $\left\{B_{1} \cap T, \ldots, B_{n} \cap T\right\}$ is an $s$-sequence). By construction $\bigcap\left\{A_{n}^{\prime}: n \in \mathbb{N}\right\} \cap R=\bigcap\left\{A_{n}: n \in \mathbb{N}\right\} \cap R$. Hence, it follows that $\alpha$ wins each play where $\bigcap\left\{A_{n}^{\prime}: n \in \mathbb{N}\right\} \cap R \neq \emptyset$.

In [11] Stegall calls a Baire space $T$ a complete Baire space, if it is regular, and contains as a dense subspace, a strongly countably complete space. Hence from Corollary 1.12. Part (iii) and Proposition 1.13, we see that complete Baire spaces are conditionally $v-\alpha$ favourable, and so $\nu-\beta$ défavourable. From this it follows that Theorem 1.10 does indeed subsume both Theorem 2 in [10] and Theorem 5 in [11].

\section{A DIFFERENTIABILITY RESULT FOR CONVEX FUNCTIONS DEFINED ON DUAL BANACH SPACES}

We say that a real-valued function $\phi$ defined on a non-empty open subset $A$ of a normed linear space $X$ is Fréchet differentiable at $x \in A$ if there exists a linear 
functional $f$ on $X$ such that for each $\varepsilon>0$ there exists a $\delta(x, \varepsilon)>0$ such that:

$$
|\phi(x+y)-\phi(x)-f(y)| \leqslant \varepsilon|y| \text { for all }|y|<\delta \text {. }
$$

In the case of a continuous convex function $\phi$ is Fréchet differentiable at $x \in A$ if and only if

$$
\lim _{\lambda \rightarrow 0} \frac{\phi(x+\lambda y)-\phi(x)}{\lambda}
$$

exists, and is approached uniformly for all $y \in S(X)$. Note, this characterisation does not hold in general. Furthermore, for convex functions we can completely determine their differentiability properties in terms of the following set-valued mapping.

The subdifferential mapping $x \rightarrow \partial \phi(x)$ associated with the convex function $\phi$ is defined by

$$
\partial \phi(x) \equiv\left\{f \in X^{*}: f(y)-f(x) \leqslant \phi(y)-\phi(x) \text { for all } y \in A\right\}
$$

It is well-known that for each $x \in A, \partial \phi(x)$ is non-empty and weak * compact, moreover it is also known that the subdifferential mapping is weak * upper semi-continuous on $A$, $[8$, p.19]. The fact that the results from Section 1 enable us to deduce differentiability results follows from the fact that a conver function $\phi$ is Fréchet differentiable at a point $x_{0} \in A$ if and only if there exists a selection $\sigma: A \rightarrow X^{*}$ of the subdifferential mapping $x \rightarrow \partial \phi(x)$ which is norm continuous at $x_{0},[8, p .19]$.

We say that a Banach space $X$ is a dual differentiability space (or $D D$ space, for short) if each continuous convex function $\phi$ defined on a non-empty subset $A$ of $X^{*}$ is Fréchet differentiable at each point of a dense and $G_{\delta}$ subset of $A$, whenever the set $\{f \in A: \partial \phi(f) \cap \widehat{X} \neq \emptyset\}$ is residual in $A$. This class of Banach spaces is very large, in fact currently, there is no known example of a Banach space which fails to be a $D D$ space. Hence it is not unreasonable to conjecture that all Banach spaces are in fact $D D$ spaces. However, a proof or counter example to this conjecture remains elusive. In this paper we content outselves with showing that if $\phi$ is a continuous convex function defined on the dual of a Banach space $X$ and $\left\{f \in X^{*}: \partial \phi(f) \cap \widehat{X} \neq \emptyset\right\}=X^{*}$, then $\phi$ is Fréchet differentiable on a dense and $G_{\delta}$ subset of $X^{*}$.

For a continuous convex function $h$ defined on a non-empty open convex subset $A$ of a normed linear space $X$, we define epi $(h) \equiv\{(x, r) \in A \times \mathbb{R}: r \geqslant h(x), x \in A\}$. It is not difficult to see that int $(\operatorname{epi}(h))=\{(x, r) \in A \times \mathbb{R}: r>h(x), x \in A\} \neq \emptyset$.

Proposition 2.1. Let $H$ be a continuous convex function defined on the dual of a Banach space $X$. Then $F \in \partial h\left(f_{0}\right)$ if and only if $(F,-1) \in X^{* *} \times \mathbb{R}$ supports epi $(h)$ at $\left(f_{0}, h\left(f_{0}\right)\right)$.

Theorem 2.2. Let $H$ be a continuous convex function defined on the dual of a Banach space $X$. If $\partial h(f) \cap \widehat{X} \neq \emptyset$ for each $f \in X^{*}$, then $\partial h\left(X^{*}\right) \subseteq \widehat{X}$. 
Proof: We may suppose without loss of generality, that $h(0)=-1$. Let $p$ be the Minkowski functional defined on $X^{*} \times \mathbb{R}$ associated with the convex set epi $(h)$. Since $h(0)=-1,(0,0) \in \operatorname{int}($ epi $(h))$ and so $p$ is well-defined and continuous on $X^{*} \times \mathbb{R}$. Consider the Banach space $X \times \mathbb{R}$ under any norm which restricts to the original norm on the subspace $X$; for instance $\|(x, r)\|=\|x\|+|r|$. Also consider the natural mapping $T:(X \times \mathbb{R})^{*} \rightarrow X^{*} \times \mathbb{R}$ defined by: $T(g)=(f, r)$ where $f(x) \equiv g(x, 0)$ and $r \equiv g(0,1) . T$ is an isomorphism from $(X \times \mathbb{R})^{*}$ onto $X^{*} \times \mathbb{R}$. Let $p^{*} \equiv p \circ T$. The following facts are readily verified.

(i) $p^{*}$ is a non-negative continuous sublinear functional on $(X \times \mathbb{R})^{*}$, and hence $p^{*}$ is the Minkowski functional associated with the closed and convex set $H \equiv\left\{g \in(X \times \mathbb{R})^{*}: p^{*}(g) \leqslant 1\right\}$;

(ii) $T(H)=$ epi $(h)$;

(iii) $\partial H=\left\{g \in(X \times \mathbb{R})^{*}: p^{*}(g)=1\right\}$ and $T(\partial H)=\partial \operatorname{epi}(h)=\{(f, h(f))$ : $\left.f \in X^{*}\right\}$;

(iv) For each $g \in \partial H, \partial p^{*}(g) \cap(\widehat{X \times \mathbb{R}}) \neq \emptyset$.

This follows from Proposition 2.1 and the fact that for each $g \in \partial H$

$$
\partial p^{*}(g)=\left\{F \in(X \times \mathbb{R})^{* *}: F(g)=p^{*}(g)=\sup \{F(f): f \in H\}\right\} .
$$

Let $H_{0} \equiv\{(x, r) \in X \times \mathbb{R}: g(x, r) \leqslant 1$ for all $g \in H\}$. It is not too difficult to see that $H_{0}$ is bounded in $X \times \mathbb{R}$. We shall show that $H_{0}$ is in fact weak compact in $X \times \mathbb{R}$. We do this via James' characterisation of weak compactness [5]. To this end, let $g \in(X \times \mathbb{R})^{*}$. We consider two cases.

(a) If $p^{*}(g)=0$, then for each $\lambda>0 p^{*}(\lambda g)=0$, and so $\lambda g \in H$. However, this means that if $(x, r) \in H_{0}$, then for each $\lambda>0, \lambda g(x, r) \leqslant 1$, which implies that $g(x, r) \leqslant 0$. Hence $g(0,0)=\max \left\{g(x, r):(x, r) \in H_{0}\right\}=$ 0 .

(b) If $p^{*}(g)>0$, then for some $\lambda_{0}>0, p^{*}\left(\lambda_{0} g\right)=1$, and so from (iv) above there exists an $(\widehat{x, r}) \in \partial p^{*}\left(\lambda_{0} g\right)$ such that $1=p^{*}\left(\lambda_{0} g\right)=$ $\lambda_{0} g(x, r)=\sup \{f(x, r): f \in H\}$. Therefore $(x, r) \in H_{0}$ and $g(x, r)=$ $\max \left\{g(y, s):(y, s) \in H_{0}\right\}$.

Hence in either case $g$ attains its maximum value on $H_{0}$, which shows that $H_{0}$ is weak compact. However, it is well known that

$$
\partial p^{*}\left((X \times \mathbb{R})^{*}\right) \subseteq H^{0} \equiv\left\{F \in(X \times \mathbb{R})^{* *}: F(g) \leqslant 1 \text { for all } g \in H\right\},
$$

and that $B^{0}=$ weak $^{*}$ - $\operatorname{cl} \widehat{H}_{0}$. Therefore we may conclude that $\partial p^{*}\left((X \times \mathbb{R})^{*}\right) \subseteq$ $(\widehat{X \times \mathbb{R}})$. We complete the proof by observing that this result in conjunction with Proposition 2.1 implies that for each $f \in X^{*}, \partial h(f) \subseteq \widehat{X}$. 
CoRollary 2.3. If $\phi$ is a continuous convex function defined on the dual of a Banach space $X$ and $\left\{f \in X^{*}: \partial \phi(f) \cap \hat{X} \neq \emptyset\right\}=X^{*}$, then $\phi$ is Fréchet differentiable on a dense and $G_{6}$ subset of $X^{*}$.

Proof: The subdifferential mapping $f \rightarrow \partial \phi(f)$ is weak ${ }^{*}$ upper semi-continuous and weak ${ }^{*}$ compact-valued on $X^{*}$. However, from Theorem 2.2 we have that $\partial \phi\left(X^{*}\right) \subseteq$ $\widehat{X}$, and so the subdifferential mapping $f \rightarrow \partial \phi(f)$ is weak upper semi-continuous and weak compact-valued on $X^{*}$. Therefore from Theorem 1.10 the subdifferential mapping admits a selection $\sigma$ which is norm continuous at each point of a dense and $G_{\delta}$ subset of $X^{*}$. The result now follows from our discussion at the start of this section.

REMARK 2.4. Actually, from Theorem 1.6 we have that the subdifferential mapping $f \rightarrow \partial \phi(f)$ admits a Baire 1 selection which is necessarily norm continuous at each point of a dense and $G_{\delta}$ subset of $X^{*}$.

We conclude this paper with the following question.

If $\phi$ is a continuous convex function defined on a non-empty open convex subset $A$ of the dual of a Banach space $X$ and $A=\{f \in A: \partial \phi(f) \cap \widehat{X} \neq \emptyset\}$, is $\phi$ necessarily Fréchet differentiable on a dense and $G_{\delta}$ subset of $A$ ?

\section{APPENDIX}

Notation. For a Banach space $X$ we shall denote by:

$\begin{array}{ll}B(X) & \{x \in X:|X| \leqslant 1\} \\ S(X) & \{x \in X:|x|=1\} \\ X^{*} & \text { the dual of } X \\ X^{* *} & \text { the second dual of } X \\ \widehat{X} & \text { the natural embedding of } X \text { into } X^{* *} \\ \operatorname{co} A & \text { the convex hull of the } A \\ \overline{\operatorname{co}} A & \text { the closed convex hull of the set } A \\ \operatorname{dist}(A, B) & \text { the number inf }\{\|a-b\|: a \in A, b \in B\}, A \neq \emptyset \text { and } B \neq \emptyset .\end{array}$

For a topological space $(T, \tau)$ we shall denote by:

$\begin{array}{ll}\text { int } A & \text { the interior of the set } A \\ \tau-\operatorname{cl} A & \text { the closure of the set } A \text { in } T \\ \partial A & \text { the boundary of the set } A\end{array}$

NOTE ADDED IN PROOF. The author has recently proven the following result: "Let $F$ be an infinite bounded subset of a Banach space $X$. Then there exists a countably infinite subset $E$ of $F$ such that $\overline{\mathrm{co}}^{\sigma_{e}} E \subseteq \overline{\mathrm{co}} F$ ". It now follows that all the results 
from Section 1 of this paper remain valid if we define partial $\sigma_{e}$-upper semi-continuity as follows.

We say that the a set-valued mapping $\Phi$ from a topological space $T$ into nonempty subsets of a Banach space $X$ is partially $\sigma_{\mathrm{e}}$-upper semi-continuous at a point $t_{0} \in T$ (or partially $\sigma_{e}$-usc, for short), if for each $\sigma_{e}$-closed, bounded and convex subset $C$ of $X$ there exists an open neighbourhood $U$ of $t_{0}$ such that $\Phi(U) \cap C=\emptyset$, whenever $\operatorname{dist}\left(\Phi\left(t_{0}\right), C\right)>0$.

\section{REFERENCES}

[1] J. Bourgain and M. Talagrand, 'Compacité extrémale', Proc. Amer. Math. Soc. 80 (1980), 68-70.

[2] G. Choquet, Lectures on analysis 1 (Benjamin, New York and Amsterdam, 1969).

[3] J.P.R. Christensen, 'Joint continuity of separately continuous functions', Proc. Amer. Math. Soc. 82 (1981), 455-461.

[4] J.R. Giles, D.A. Gregory and B. Sims, 'Geometrical implications of upper semi-continuity of the duality mapping on a Banach space', Pacific J. Math. 79 (1978), 99-109.

[5] R.C. James, 'Reflexivity and the supremum of linear functionals', Israel J. Math. 13 (1972), 289-230.

[6] S.S. Kurana, 'Pointwise compactness on extreme points', Proc. Amer. Math. Soc. 83 (1981), 347-348.

[7] I. Namioka, 'Separate continuity and joint continuity', Pacific J. Math. 51 (1974), 515-531.

[8] R.R. Phelps, 'Convex functions, monotone operators and differentiability', Lecture Notes in Mathematics 1364 (Springer-Verlag, Berlin, Heidelberg, New York, 1993).

[8] J. Saint-Raymond, 'Jeux topologique et espaces de Namioka', Proc. Amer. Math. Soc. 87 (1983), 499-504.

[10] C. Stegall, 'Generalizations of a theorem of Namioka', Proc. Amer. Math. Soc. 102 (1988), 559-564.

[11] C. Stegall, 'Functions of the first Baire class with values in Banach spaces', Proc. Amer. Math. Soc. 111 (1991), 981-991.

Department of Mathematics

The University of Auckland

Private Bag 92019

Auckland

New Zealand 